\title{
A Robust Algorithm for Characterizing Anisotropic Local Structures
}

\author{
Kazunori Okada ${ }^{1}$, Dorin Comaniciu ${ }^{1}$, Navneet Dalal $^{2}$, and Arun Krishnan ${ }^{3}$ \\ 1 Real-Time Vision \& Modeling Department \\ Siemens Corporate Research, Inc. \\ 755 College Road East, Princeton, NJ 08540, USA \\ 2 INRIA Rhône-Alpes \\ 655, avenue de l'Europe 38330 Montbonnot, France \\ 3 CAD Program \\ Siemens Medical Solutions USA, Inc. \\ 51 Valley Stream Parkway, Malvern, PA 19355, USA
}

\begin{abstract}
This paper proposes a robust estimation and validation framework for characterizing local structures in a positive multi-variate continuous function approximated by a Gaussian-based model. The new solution is robust against data with large deviations from the model and margin-truncations induced by neighboring structures. To this goal, it unifies robust statistical estimation for parametric model fitting and multi-scale analysis based on continuous scale-space theory. The unification is realized by formally extending the mean shift-based density analysis towards continuous signals whose local structure is characterized by an anisotropic fully-parameterized covariance matrix. A statistical validation method based on analyzing residual error of the chi-square fitting is also proposed to complement this estimation framework. The strength of our solution is the aforementioned robustness. Experiments with synthetic 1D and 2D data clearly demonstrate this advantage in comparison with the $\gamma$-normalized Laplacian approach 12 and the standard sample estimation approach [13 p.179]. The new framework is applied to 3D volumetric analysis of lung tumors. A 3D implementation is evaluated with high-resolution CT images of 14 patients with 77 tumors, including 6 part-solid or ground-glass opacity nodules that are highly nonGaussian and clinically significant. Our system accurately estimated 3D anisotropic spread and orientation for $82 \%$ of the total tumors and also correctly rejected all the failures without any false rejection and false acceptance. This system processes each 32-voxel volume-of-interest by an average of two seconds with a $2.4 \mathrm{GHz}$ Intel CPU. Our framework is generic and can be applied for the analysis of blob-like structures in various other applications.
\end{abstract}

\section{Introduction}

This paper presents a robust estimation and validation framework for characterizing a $d$-variate positive function that can be locally approximated by a 
Gaussian-based model. Gaussian model fitting is a well-studied standard technique [4, ch.2]. However, it is not trivial to fit such a model to data with outliers and margin-truncation induced by neighboring structures. For example, minimum volume ellipsoid covariance estimator [17] addresses the robustness to the outliers however its effectiveness is limited regarding the truncation issue. Fig.1 illustrates our problem with some real medical imaging examples of lung tumors in 3D CT data. The figure shows 2D dissections and 1D profiles of two tumors. The symbol $\mathbf{x}$ and solid-line ellipses denote our method's estimates. In developing an algorithm to describe the tumors, our solution must be robust against 1) influences from surrounding structures (i.e., margin-truncation: Fig 1a,b), 2) deviation of the signal from a Gaussian model (i.e., non-Gaussianity: Fig [1 c,d), and 3) uncertainty in the given marker location (i.e., initialization: Fig[1a,c).

Our proposed solution unifies robust statistical methods for density gradient estimation [3] and continuous linear scale-space theory [21,9,12]. By likening the arbitrary positive function describing an image signal to the probability density function, the mean shift-based analysis is further developed towards 1) Gaussian model fitting to a continuous positive function and 2) anisotropic fully-parameterized covariance estimation. Its robustness is due to the multiscale nature of this framework that implicitly exploits the scale-space function. A statistical validation method based on chi-square analysis is also proposed to complement this robust estimation framework. Sections 2 and 3 formally describe our solution. The robustness is empirically studied with synthetic data and the results are described in Section 4.1.

\subsection{Medical Imaging Applications}

One of the key problems in the volumetric medical image analysis is to characterize the 3D local structure of tumors across various scales. The size and shape of tumors vary largely in practice. Such underlining scales of tumors also provide important clinical information, correlating highly with probability of malignancy. A large number of studies have been accumulated for automatic detection and characterization of lung nodules [19]. Several recent studies (e.g., 10,18]) exploited 3D information of nodules provided in X-ray computed-tomography (CT) images. However, these methods, based on the template matching technique, assumed the nodules to be spherical. Recent clinical studies suggested that part- and non-solid or ground-glass opacity (GGO) nodules, whose shape deviates largely from such a spherical model (Fig 1k, d), are more likely to be malignant than solid ones 6 . One of our motivations of this study is to address this clinical demand by considering the robust estimation of $3 \mathrm{D}$ tumor spread and orientation with non-spherical modeling. We evaluate the proposed framework applied for the pulmonary CT data in Section 4.2. 


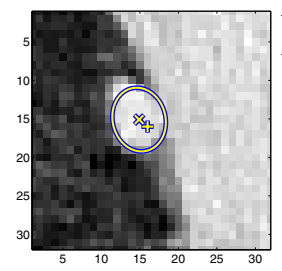

(a)

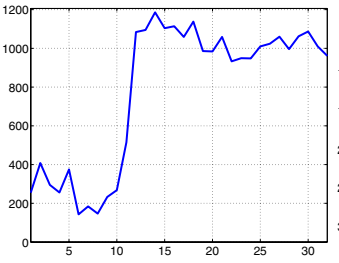

(b)

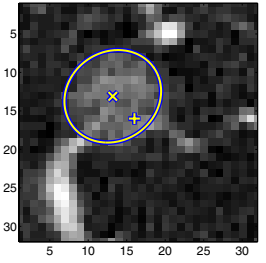

(c)

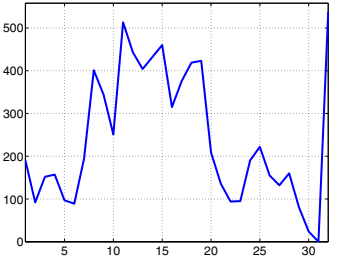

(d)

Fig. 1. An illustration of our problem with lung tumor examples captured in 3D CT data. From left to right, (a): on-the-wall tumor in 2D dissection, (b): 1D horizontal profile of (a) through the tumor center, (c): non-solid (GGO) tumor, and (d): 1D vertical profile of (c). "+" denotes markers used as initialization points provided by expert radiologists. Our method's estimates of the tumor center and anisotropic spread are shown by " $\mathrm{x}$ " and $50 \%$ confidence ellipses, respectively.

\section{Multi-scale Analysis of Local Structure}

\subsection{Signal Model}

Given a $d$-dimensional continuous signal $f(\mathbf{x})$ with non-negative values, we use the symbol $\mathbf{u}$ for describing the location of a spatial local maximum of $f$ (or a mode in the sense of density estimation). Suppose that the local region of $f$ around $\mathbf{u}$ can be approximated by a product of a $d$-variate Gaussian function and a positive multiplicative parameter,

$$
\begin{aligned}
f(\mathbf{x}) & \simeq \alpha \times\left.\Phi(\mathbf{x} ; \mathbf{u}, \mathbf{\Sigma})\right|_{\mathbf{x} \in \mathcal{S}} \\
\Phi(\mathbf{x} ; \mathbf{u}, \mathbf{\Sigma}) & =(2 \pi)^{-d / 2}|\mathbf{\Sigma}|^{-1 / 2} \exp \left(-\frac{1}{2}(\mathbf{x}-\mathbf{u})^{t} \mathbf{\Sigma}^{-1}(\mathbf{x}-\mathbf{u})\right)
\end{aligned}
$$

where $\mathcal{S}$ is a set of data points in the neighborhood of $\mathbf{u}$, belonging to the basin of attraction of $\mathbf{u}$. An alternative is to consider a model with a DC component $\beta \geq 0$ so that $f \simeq \alpha \times \Phi+\beta$. It is, however, straightforward to locally offset the DC component. Thus we will not consider it within our estimation framework favoring a simpler form. Later, we will revisit this extended model for the statistical validation of the resulting estimates. The problem of our interest can now be understood as the parametric model fitting and the estimation of the model parameters: mean $\mathbf{u}$, covariance $\boldsymbol{\Sigma}$, and amplitude $\alpha$. The mean and covariance of $\Phi$ describe the spatial local maximum and spread of the local structure, respectively. The anisotropy of such structure can be specified only by a fully-parameterized covariance.

\subsection{Scale-Space Representation}

The scale-space theory [21912] states that, given any $d$-dimensional continuous signal $f: \mathcal{R}^{d} \rightarrow \mathcal{R}$, the scale-space representation $F: \mathcal{R}^{d} \times \mathcal{R}_{+} \rightarrow \mathcal{R}$ of $f$ is 
defined to be the solution of the diffusion equation, $\partial_{h} F=1 / 2 \nabla^{2} F$, or equivalently the convolution of the signal with Gaussian kernels $\Phi(\mathbf{x} ; \mathbf{0}, \mathbf{H})$ of various bandwidths (or scales) $\mathbf{H} \in \mathcal{R}^{d \times d}$,

$$
F(\mathbf{x} ; \mathbf{H})=f(\mathbf{x}) * \Phi(\mathbf{x} ; \mathbf{0}, \mathbf{H}) .
$$

When $\mathbf{H}=h \mathbf{I}(h>0), F$ represents the solution of the isotropic diffusion process 12 and also the Tikhonov regularized solution of a functional minimization problem, assuming that scale invariance and semi-group constraints are satisfied [14]. When $\mathbf{H}$ is allowed to be a fully parameterized symmetric positive definite matrix, $F$ represents the solution of an anisotropic homogeneous diffusion process $\partial_{\mathbf{H}} F=1 / 2 \nabla \nabla^{t} F$ that is related, but not equivalent, to the well-known anisotropic diffusion [15].

\subsection{Mean Shift Procedure for Continuous Scale-Space Signal}

In this section, we further develop the fixed-bandwidth mean shift [2], introduced previously for the non-parametric point density estimation, towards the analysis of continuous signal evaluated in the linear scale-space.

The gradient of the scale-space representation $F(\mathbf{x} ; \mathbf{H})$ can be written as convolution of $f$ with the DOG kernel $\nabla \Phi$, since the gradient operator commutes across the convolution operation. Some algebra reveals that $\nabla F$ can be expressed as a function of a vector whose form resembles the density mean shift,

$$
\begin{aligned}
\nabla F(\mathbf{x} ; \mathbf{H}) & =f(\mathbf{x}) * \nabla \Phi(\mathbf{x} ; \mathbf{H}) \\
& =\int f\left(\mathbf{x}^{\prime}\right) \Phi\left(\mathbf{x}-\mathbf{x}^{\prime} ; \mathbf{H}\right) \mathbf{H}^{-1}\left(\mathbf{x}^{\prime}-\mathbf{x}\right) d \mathbf{x}^{\prime} \\
& =\mathbf{H}^{-1} F(\mathbf{x} ; \mathbf{H}) \mathbf{m}(\mathbf{x} ; \mathbf{H}) \\
\mathbf{m}(\mathbf{x} ; \mathbf{H}) & \equiv \frac{\int \mathbf{x}^{\prime} \Phi\left(\mathbf{x}-\mathbf{x}^{\prime} ; \mathbf{H}\right) f\left(\mathbf{x}^{\prime}\right) d \mathbf{x}^{\prime}}{\int \Phi\left(\mathbf{x}-\mathbf{x}^{\prime} ; \mathbf{H}\right) f\left(\mathbf{x}^{\prime}\right) d \mathbf{x}^{\prime}}-\mathbf{x} .
\end{aligned}
$$

Eq. (5) defines the extended fixed-bandwidth mean shift vector for $f$. Setting $f\left(\mathbf{x}^{\prime}\right)=1$ in Eq. (5) results in the same form as the density mean shift vector. Note however that $\mathbf{x}$ in Eq. (5) is an ordinal variable while a random variable was considered in [2]. Eq.(5]) can be seen as introducing a weight variable $w \equiv f\left(\mathbf{x}^{\prime}\right)$ to the kernel $\Phi\left(\mathbf{x}-\mathbf{x}^{\prime}\right)$. Therefore, an arithmetic mean of $\mathbf{x}^{\prime}$ in our case is not weighted by the Gaussian kernel but by its product with the signal $\Phi\left(\mathbf{x}-\mathbf{x}^{\prime}\right) f\left(\mathbf{x}^{\prime}\right)$.

The mean shift procedure [3] is defined as iterative updates of a data point $\mathbf{x}_{i}$ until its convergence at $\mathbf{y}_{i}^{m}$,

$$
\mathbf{y}_{j+1}=\mathbf{m}\left(\mathbf{y}_{j} ; \mathbf{H}\right)+\mathbf{y}_{j} ; \mathbf{y}_{0}=\mathbf{x}_{i} .
$$

Such iteration gives a robust and efficient algorithm of gradient-ascent, since $\mathbf{m}(\mathbf{x} ; \mathbf{H})$ can be interpreted as a normalized gradient by rewriting Eq.(4); $\mathbf{m}(\mathbf{x} ; \mathbf{H})=\mathbf{H} \nabla F(\mathbf{x} ; \mathbf{H}) / F(\mathbf{x} ; \mathbf{H}) . F$ is strictly non-negative valued since $f$ is assumed to be non-negative. Therefore, the direction of the mean shift vector aligns with the exact gradient direction when $\mathbf{H}$ is isotropic with a positive scale. 


\subsection{Finding Spatial Local Maxima}

We assume that the signal is given with information of where the target structure is roughly located but we do not have explicit knowledge of its spread. The marker point $\mathbf{x}_{p}$ indicates such location information. We allow $\mathbf{x}_{p}$ to be placed anywhere within the basin of attraction $\mathcal{S}$ of the target structure. To increase the robustness of this approach, we run $N_{1}$ mean shift procedures initialized by sampling the neighborhood of $\mathbf{x}_{p}$ uniformly. The majority of the procedure's convergence at the same location indicates the location of the maximum. The point proximity is defined by using the Mahalanobis distance with $\mathbf{H}$. This approach is efficient because it does not require the time-consuming explicit construction of $F(\mathbf{x} ; \mathbf{H})$ from $f(\mathbf{x})$.

\subsection{Robust Anisotropic Covariance Estimation by Constrained Least-Squares in the Basin of Attraction}

In the sequel we estimate the fully parameterized covariance matrix $\boldsymbol{\Sigma}$ in Eq.(1), characterizing the $d$-dimensional anisotropic spread and orientation of the signal $f$ around the local maximum $\mathbf{u}$. Classical scale-space approaches relying on the $\gamma$-normalized Laplacian [12] are limited to the isotropic case thus not applicable to this problem. Another approach is the standard sample estimation of $\boldsymbol{\Sigma}$ by treating $f$ as a density function [13, p.179]. However, this approach becomes suboptimal in the presence of the margin-truncations. Addressing this issue, we present a constrained least-squares framework for the estimation of the anisotropic fully-parameterized covariance of interest based on the mean shift vectors collected in the basin of attraction of $\mathbf{u}$.

With the signal model of Eq.(1), the definition of the mean shift vector of Eq. (5) can be rewritten as a function of $\boldsymbol{\Sigma}$,

$$
\begin{aligned}
\mathbf{m}\left(\mathbf{y}_{j} ; \mathbf{H}\right) & =\mathbf{H} \frac{\nabla F\left(\mathbf{y}_{j} ; \mathbf{H}\right)}{F\left(\mathbf{y}_{j} ; \mathbf{H}\right)} \\
& \simeq \mathbf{H} \frac{\alpha \Phi\left(\mathbf{y}_{j} ; \mathbf{u}, \boldsymbol{\Sigma}+\mathbf{H}\right)(\boldsymbol{\Sigma}+\mathbf{H})^{-1}\left(\mathbf{u}-\mathbf{y}_{j}\right)}{\alpha \Phi\left(\mathbf{y}_{j} ; \mathbf{u}, \boldsymbol{\Sigma}+\mathbf{H}\right)} \\
& =\mathbf{H}(\boldsymbol{\Sigma}+\mathbf{H})^{-1}\left(\mathbf{u}-\mathbf{y}_{j}\right) .
\end{aligned}
$$

Further rewriting Eq.(7) results in a linear matrix equation of unknown $\boldsymbol{\Sigma}$,

$$
\boldsymbol{\Sigma} \mathbf{H}^{-1} \mathbf{m}_{j}=\mathbf{b}_{j}
$$

where $\mathbf{m}_{j} \equiv \mathbf{m}\left(\mathbf{y}_{j} ; \mathbf{H}\right)$ and $\mathbf{b}_{j} \equiv \mathbf{u}-\mathbf{y}_{j}-\mathbf{m}_{j}$.

An over-complete set of the linear equations can be formed by using all the trajectory points $\left\{\mathbf{y}_{j} \mid j=1, . ., t_{u}\right\}$ located within the basin of attraction $\mathcal{S}$. For efficiently collecting a sufficient number of samples $\left\{\left(\mathbf{y}_{j}, \mathbf{m}_{j}\right)\right\}$, we run $N_{2}$ mean shift procedures initialized by sampling the neighborhood of $\mathbf{u}$ uniformly. This results in $t_{u}$ samples $\left(t_{u}=\sum_{i=1}^{N_{2}} t_{i}\right)$, where $t_{i}$ denotes the number of points 
on the trajectory starting from $x_{i}$. The system described in Eq.(8) is solved by considering the following constrained least-squares problem [75],

$$
\begin{aligned}
\mathbf{A} \boldsymbol{\Sigma} & =\mathbf{B} \\
\boldsymbol{\Sigma} & \in \mathcal{S P} \mathcal{D} \\
\mathbf{A} & =\left(\mathbf{m}_{1}, . ., \mathbf{m}_{t_{u}}\right)^{t} \mathbf{H}^{-t} \\
\mathbf{B} & =\left(\mathbf{b}_{1}, . ., \mathbf{b}_{t_{u}}\right)^{t}
\end{aligned}
$$

where $\mathcal{S P D}$ denotes a set of symmetric positive definite matrices in $\mathcal{R}^{d \times d}$.

Following [1, the unique solution $\boldsymbol{\Sigma}^{*}$ of Eq.(9) is expressed by,

$$
\boldsymbol{\Sigma}^{*}=\mathbf{U}_{P} \boldsymbol{\Sigma}_{P}^{-1} \mathbf{U}_{\tilde{Q}} \boldsymbol{\Sigma}_{\tilde{Q}} \mathbf{U}_{\tilde{Q}}^{t} \boldsymbol{\Sigma}_{P}^{-1} \mathbf{U}_{P}^{t}
$$

which involves symmetric Schur decompositions [5, p.393] of the matrices $\mathbf{P} \equiv$ $\mathbf{A}^{t} \mathbf{A}$ and $\tilde{\mathbf{Q}} \equiv \boldsymbol{\Sigma}_{P} \mathbf{U}_{P}^{t} \mathbf{Q} \mathbf{U}_{P} \boldsymbol{\Sigma}_{P}$ given $\mathbf{Q} \equiv \mathbf{B}^{t} \mathbf{B}$, i.e.,

$$
\begin{aligned}
& \mathbf{P}=\mathbf{U}_{P} \boldsymbol{\Sigma}_{P}^{2} \mathbf{U}_{P}^{t} \\
& \tilde{\mathbf{Q}}=\mathbf{U}_{\tilde{Q}} \boldsymbol{\Sigma}_{\tilde{Q}}^{2} \mathbf{U}_{\tilde{Q}}^{t} .
\end{aligned}
$$

The solution $\boldsymbol{\Sigma}^{*}$ is derived from finding $\mathbf{Y}^{*}$ in the Cholesky factorization of $\boldsymbol{\Sigma}=\mathbf{Y} \mathbf{Y}^{t}$. It can be shown that $\boldsymbol{\Sigma}^{*}$ uniquely minimizes an area criterion $\| \mathbf{A Y}-$ $\mathbf{B Y} \mathbf{Y}^{-t} \|_{F}^{2}$ where \|\|$_{F}$ denotes the Frobenius norm. This area criterion is related to the total least-squares [20] since errors in both $\mathbf{A}$ and $\mathbf{B}$ are considered for the minimization.

\subsection{Scale Selection Criterion}

The multi-scale analysis treats $\mathbf{H}$ as a variable parameter. It is supposed that a set of analysis bandwidths $\left\{\mathbf{H}_{k} \mid k=1, \ldots, K\right\}$ is given a priori. Our scale selection criterion is based on the stability test [2]. Given a set of estimates $\left\{\left(\mathbf{u}_{k}, \boldsymbol{\Sigma}_{k}\right)\right\}$ for a series of the successive analysis bandwidths, a form of the Jensen-Shannon divergence is defined by,

$$
J S(k)=\frac{1}{2} \log \frac{\left|\frac{1}{2 a+1} \sum_{i=k-a}^{k+a} \boldsymbol{\Sigma}_{i}\right|}{\sqrt[2 a+1]{\prod_{i=k-a}^{k+a}\left|\boldsymbol{\Sigma}_{i}\right|}}+\frac{1}{2} \sum_{i=k-a}^{k+a}\left(\mathbf{u}_{i}-\mathbf{u}\right)^{t}\left(\sum_{i=k-a}^{k+a} \boldsymbol{\Sigma}_{i}\right)^{-1}\left(\mathbf{u}_{i}-\mathbf{u}\right)
$$

where $\mathbf{u}=\frac{1}{2 a+1} \sum_{k-a}^{k+a} \mathbf{u}_{i}$ and $a$ define the neighborhood width of the divergence computation. The most stable estimate across the analysis bandwidths provides a local minimum of the divergence profile. We treat this result as the final estimation of our multi-scale analysis.

\section{Statistical Validation}

In this section, we present a goodness-of-fit measure for validating the resulting estimates. Such statistical validation gives a principled means for rejecting 
accidental ill-estimates. We treat this problem as analysis of chi-square fitting residual errors. We employ a linear model with an additive parameter of the DC component; $f \simeq \alpha \times \Phi+\beta$. Recall that our estimation model is without the DC. The additional degree of freedom introduced serves as another goodness-offit indicator. Given the estimate pair $\left(\mathbf{u}^{*}, \mathbf{\Sigma}^{*}\right)$, the following defines the signal response estimate $\hat{f}$ with two unknowns,

$$
\hat{f}\left(\mathbf{x}, \mathbf{u}^{*}, \mathbf{\Sigma}^{*} ; \alpha, \beta\right)=\alpha \times \Phi\left(\mathbf{x} ; \mathbf{u}^{*}, \mathbf{\Sigma}^{*}\right)+\left.\beta\right|_{\mathbf{X} \in \mathcal{S}}
$$

The chi-square statistic indicates the residual error of the fitted model $\hat{f}(\mathbf{x})[16$, p.660],

$$
\chi^{2} \equiv \sum_{i \in S}\left(\frac{f\left(\mathbf{x}_{i}\right)-\hat{f}\left(\mathbf{x}_{i}\right)}{\sigma_{i}}\right)^{2}=\sum_{i \in S}\left(\frac{f\left(\mathbf{x}_{i}\right)-\alpha \Phi\left(\mathbf{x}_{i}\right)-\beta}{\sigma_{i}}\right)^{2}
$$

where $\sigma_{i}$ is local uncertainty of normally distributed error $\left(f\left(\mathbf{x}_{i}\right)-\hat{f}\left(\mathbf{x}_{i}\right)\right)^{2}$.

Parameters $\alpha$ and $\beta$ are estimated by chi-square fitting. Since both are nonnegative, we introduce parameters $a$ and $b$ such that $\alpha=a^{2}$ and $\beta=b^{2}$. The estimates $\alpha^{*}$ and $\beta^{*}$ are given by solving $\partial \chi^{2} / \partial a=0$ and $\partial \chi^{2} / \partial b=0$,

$$
\left(\alpha^{*}, \beta^{*}\right)= \begin{cases}(p, q) & \text { if } \mathrm{p}>0 \text { and } \mathrm{q}>0 \\ \left(\frac{\sum f\left(\mathbf{x}_{i}\right) \Phi\left(\mathbf{x}_{i}\right)}{\sum \Phi\left(\mathbf{X}_{i}\right)^{2}}, 0\right) & \text { if } \mathrm{p}>0 \text { and } \mathrm{q} \leq 0 \\ \left(0, \frac{\sum f\left(\mathbf{x}_{i}\right)}{N_{s}}\right) & \text { if } \mathrm{p} \leq 0 \text { and } \mathrm{q}>0 \\ (0,0) & \text { if } \mathrm{p} \leq 0 \text { and } \mathrm{q} \leq 0\end{cases}
$$

where $\sigma=\sigma_{i}$ for all $i$,

$$
\begin{aligned}
& p=\frac{N_{s} \sum f\left(\mathbf{x}_{i}\right) \Phi\left(\mathbf{x}_{i}\right)-\sum f\left(\mathbf{x}_{i}\right) \sum \Phi\left(\mathbf{x}_{i}\right)}{N_{s} \sum \Phi\left(\mathbf{x}_{i}\right)^{2}-\left(\sum \Phi\left(\mathbf{x}_{i}\right)\right)^{2}} \\
& q=\frac{\sum f\left(\mathbf{x}_{i}\right) \sum \Phi\left(\mathbf{x}_{i}\right)^{2}-\sum \Phi\left(\mathbf{x}_{i}\right) \sum f\left(\mathbf{x}_{i}\right) \Phi\left(\mathbf{x}_{i}\right)}{N_{s} \sum \Phi\left(\mathbf{x}_{i}\right)^{2}-\left(\sum \Phi\left(\mathbf{x}_{i}\right)\right)^{2}}
\end{aligned}
$$

and $N_{s}$ is the number of samples in $\mathcal{S}$ and all the summations are over $i \in S$.

Given the above parameter estimates, $\chi^{2}$ is computed by using Eq.(13). Chisquare probability function $Q[16$, p.221] is employed to indicate an ill-fit of our model to the given signal,

$$
Q\left(\chi^{2} \mid \nu\right)=Q\left(\frac{N_{s}-M}{2}, \frac{\chi^{2}}{2}\right)=g\left(\frac{N_{s}-M}{2}, \frac{\chi^{2}}{2}\right) .
$$

In Eq. (17), $g$ is the incomplete gamma function [16, ch.6.2] with the number of degrees of freedom $\nu=(N-M) / 2$, and $M$ is the number of parameters.

Finally, we obtain the following rejection criterion,

$$
\text { Reject }\left(\mathbf{u}^{*}, \boldsymbol{\Sigma}^{*}\right) \text { if } Q<t h_{1} \text { or } \beta^{*}>t h_{2} .
$$

The threshold for $Q$ is set conservatively to the common confidence level $t h_{1}=$ 0.001 [16] p.664]. Having a large estimate for $\beta$ also indicates an ill-fit with our estimation model without the DC. The threshold $t h_{2}$ for $\beta$ can be learned from training samples for specific applications. 


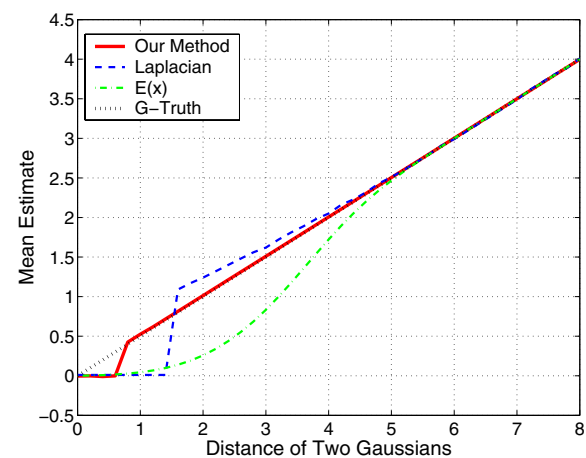

(a)

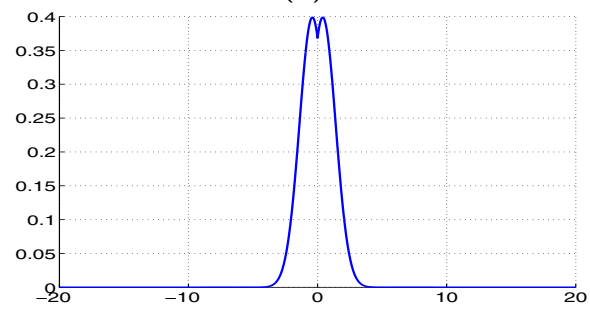

(c)

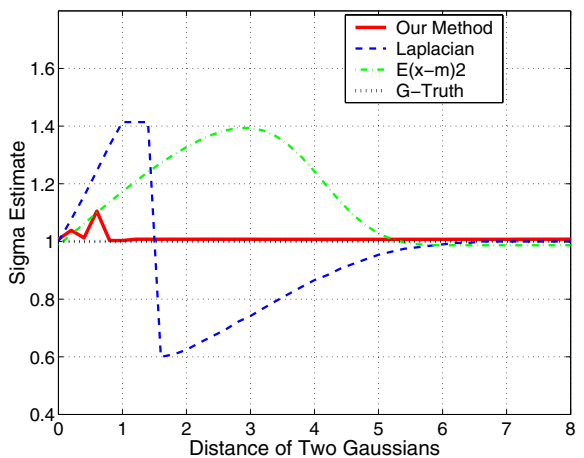

(b)

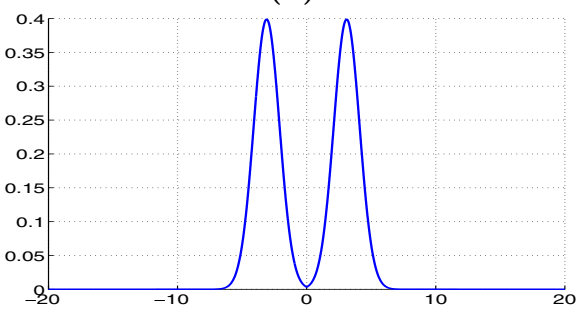

(d)

Fig. 2. Comparison of our method (solid-line) with $\gamma$-normalized Laplacian (dashedline) and standard sample estimate (dot-dashed-line) using 1D synthetic data. The ground-truth $u=D / 2$ and $\sigma=1$ are denoted by dotted-line. Test data is generated by superimposing two Gaussians with a varying distance $D$ for evaluating robustness of estimates against biases caused by neighboring structures. (a): local maxima estimates, (b): scale estimates, (c): our method's break-point $D=0.8$, below which estimations are subjected to the bias. (c): $\gamma$-normalized Laplacian's break-point $D=6.2$.

\section{Experiments}

\subsection{Synthetic Data}

The proposed framework is examined with $1 \mathrm{D}$ and $2 \mathrm{D}$ synthetic data. Fig.2 compares local maximum and scale estimates by a 1D implementation of our algorithm with those by the $\gamma$-normalized Laplacian 12 and the standard sample estimation [13, p.179]. The test data is generated at each location by taking the maximum of two superimposed 1D Gaussians offset by a varying distance $D$. Each Gaussian has the same variance $\sigma=1$ and hight $\alpha=1$. The $1 \mathrm{D}$ system employs all the available data points $\left(N_{1}=N_{2}=N_{S}\right)$ and 40 analysis scales with 0.05 interval $\left(h=\left(0.1^{2}, 0.15^{2}, . ., 2^{2}\right)\right.$ for $\left.\mathbf{H}=h \mathbf{I}\right)$. For the sample variance estimation, the densities $p\left(x_{i}\right)$ are approximated by $f\left(x_{i}\right)$ normalized by the probability mass within $\pm 1 \sigma$ around the true maximum. The results indicated that our method achieved robust and accurate estimations even with the presence of the severe margin-truncations, clearly demonstrating the advantage of our framework. Fig[3 shows examples with 2D synthetic data. Estimates, shown as 50\% 


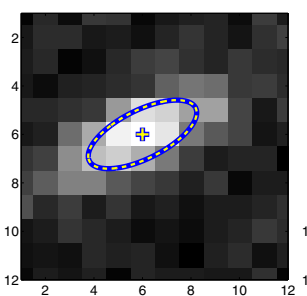

(a)

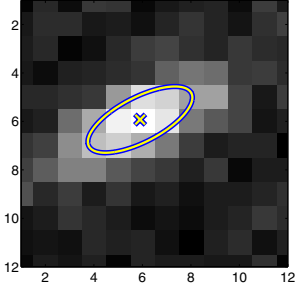

(b)

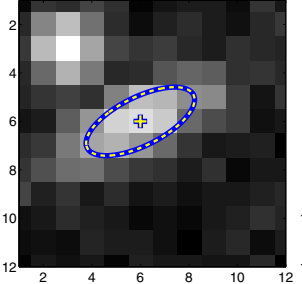

(c)

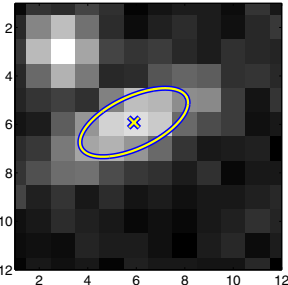

(d)

Fig. 3. Examples with $2 \mathrm{D}$ synthetic data. (a) and (b) illustrate the ground-truth and our method's estimate for an anisotropic Gaussian $\boldsymbol{\Sigma}=\left[\begin{array}{l}2-2 ;-2 \\ 5\end{array}\right]$ with random additive noise. (c) and (d) show those for two Gaussians with the noise. The center of the smaller Gaussian is deviated by 4 Mahalanobis distance away from the target Gaussian. "+" and dashed-ellipses indicate ground-truth local maximum and spread. " $\mathrm{x}$ " and solid-ellipses display those estimated by our 2D algorithm.

confidence ellipses, by a 2D implementation of our method are compared for two types of test data in the presence of random noise. This $2 \mathrm{D}$ implementation utilizes all available data points and 12 analysis scales $\left(h=\left(0.5^{2}, 0.75^{2}, . ., 3.25^{2}\right)\right)$. The results are almost identical to the ground-truth despite the presence of the random noise and the neighboring structure.

\subsection{Lung HRCT Data}

A 3D implementation of the proposed algorithm is evaluated with high-resolution computed-tomography (HRCT) images of pulmonary tumors. Each volumetric image consists of 12-bit positive values over an array of $512 \times 512$ lattices.

A straightforward implementation of our algorithm without any 3D specific adaptation provides the $3 \mathrm{D}$ tumor analysis system. A set of analysis bandwidths (18 scales with 0.25 interval $h=\left(0.50^{2}, 0.75^{2}, . ., 4.75^{2}\right)$ ) and markers indicating rough tumor locations are given to the system a priori. The marker locations are provided by expert radiologists, however most of the markers deviate from the tumor centers with a certain degree. We use uniform sampling in the 3-voxel neighborhood of the marker (i.e., $N_{1}=7$ ). The same strategy is employed for initializing the mean shift trajectories around the local maximum (i.e., $N_{2}=7$ ). The neighborhood width of the divergence computation is set to $a=1$ (considering only three adjacent scales). For the validation, all data points that lie within the $90 \%$ confidence ellipsoid of $\left(\mathbf{u}^{*}, \boldsymbol{\Sigma}^{*}\right)$ are used. The degrees of freedom in Eq.(17) are given by $M=3+6+2=11$. The $\beta$ threshold in Criterion(18) is set to $t h_{2}=400$. The global uncertainty $\sigma$ in Eq.(13) is estimated from the sample variance of 77 tumor data, resulting in $\sigma=356$. This tumor analysis system is implemented in $\mathrm{C}$ language and processes each 32-voxel volume-of-interest (VOI) by an average of two seconds with a $2.4 \mathrm{GHz}$ Intel CPU.

HRCT data of 14 patients displaying the total of 77 pulmonary tumors were used for this evaluation. 63 cases resulted in successful estimation confirmed by expert inspection. All the solitary tumors were correctly estimated. Most of the 

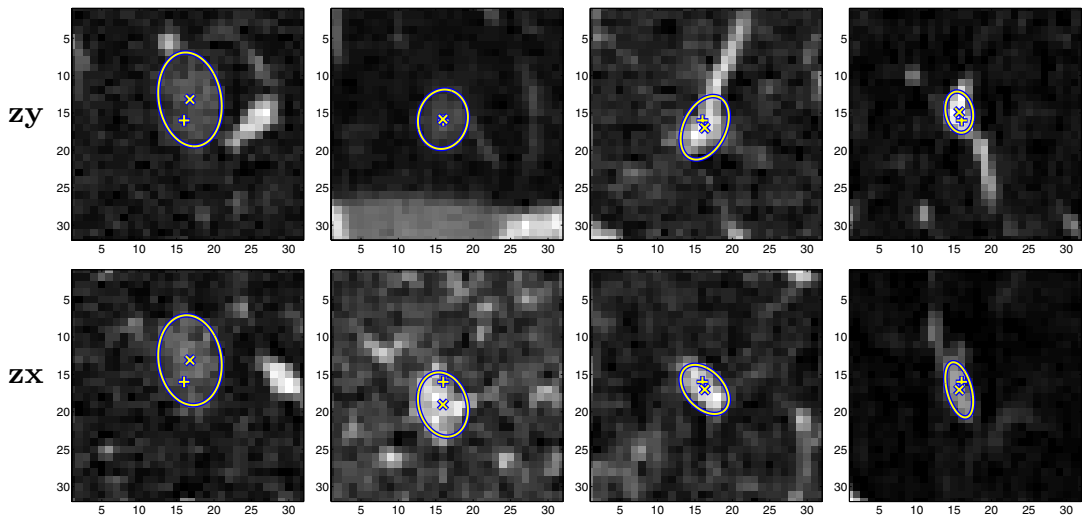

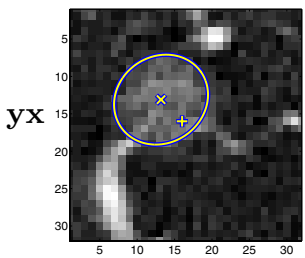

(a)
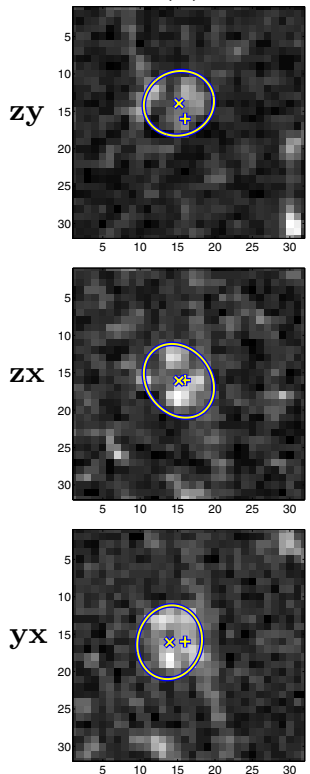

(e)

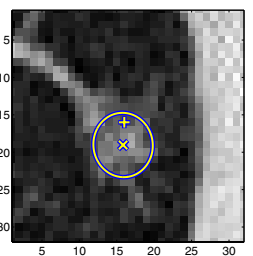

(b)
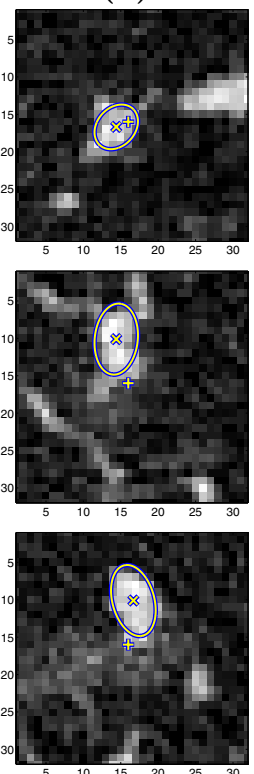

(f)

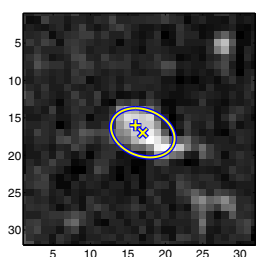

(c)
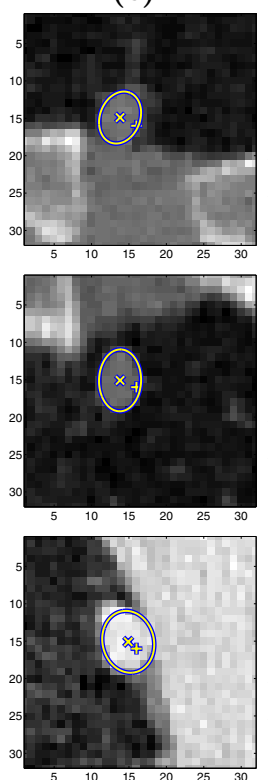

(g)

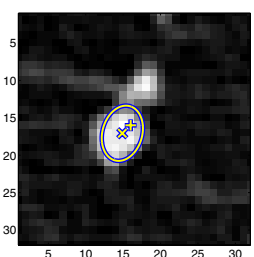

(d)
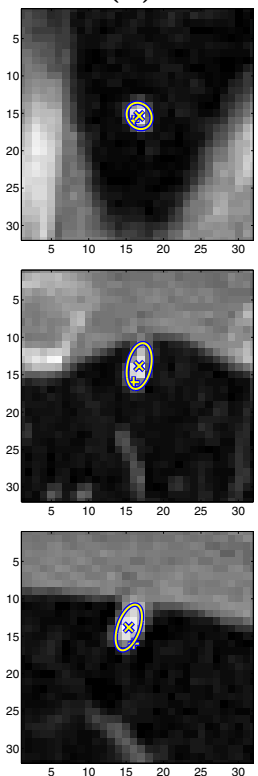

(h)

Fig. 4. Examples of the estimation results with 3D HRCT data. The marker locations are indicated by "+". The estimated local maxima are indicated by "x". The estimated spread of the tumors are shown as 2D intersections of $50 \%$ confidence ellipsoids. Cases (a) and (b) are GGO nodules identified by experts. Cases (c) to (f) are tumors with irregular non-spherical shapes. Cases $(\mathrm{g})$ and $(\mathrm{h})$ illustrate tumors on the lung wall. 


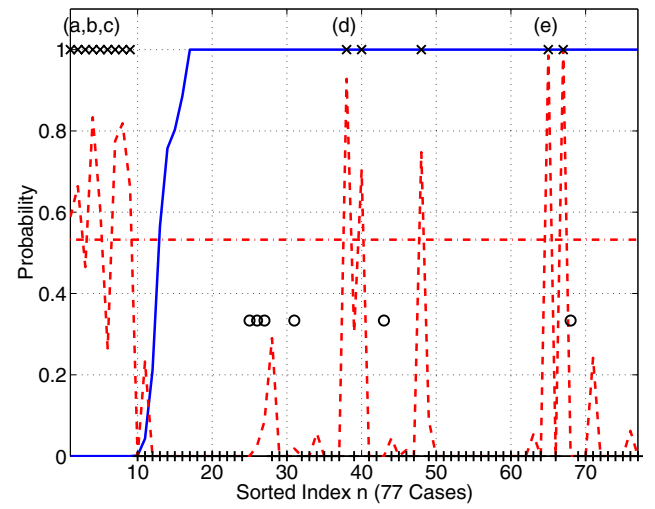

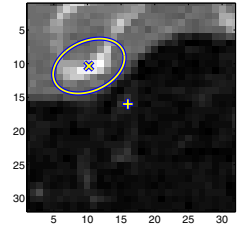

(a)

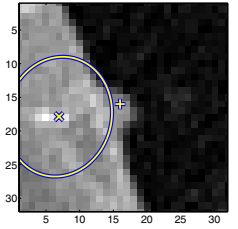

(b)

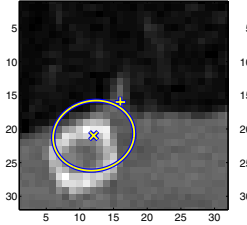

(c)

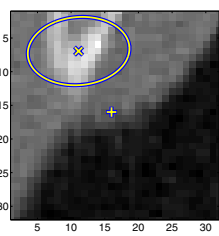

(d)

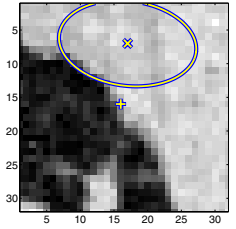

(e)

Fig. 5. Experimental results for the validation process. The top plot illustrates the $Q$ probability (solid-line) and $\beta$ estimate (dashed-line) for each test case. The symbols "+", "x", and "o" indicate correct, failure, and GGO nodule cases, respectively. $\beta$ values are normalized to fit within the range of this plot. A horizontal dashed-line indicates the $\beta$-threshold $t h_{2}=400$. The bottom images show examples of correctly rejected failures. Legend of these images are the same as Fig 4 Cases (a) and (c) satisfied the rejection conditions of both $Q$ and $\beta$ while Case (b) met only the $Q$ condition and Cases (d) and (e) met only the $\beta$ condition.

failures were due to small tumors whose shape was a partial ellipsoid located on lung walls and near rib structures. All the 14 failures were successfully rejected by the validation process without false rejection and false acceptance. The data includes six cases of the part- and non-solid or ground-glass opacity nodules (GGO nodules, see Fig[1 ,d and Fig.4a,b). All GGO nodules were successfully estimated and accepted.

Fig 4 shows examples of the resulting center and spread estimates. It illustrates cases with the irregular, GGO, and on-the-wall nodules whose geometrical shapes are largely deviated from the Gaussian structure. The correct estimations for these difficult cases demonstrate the robustness and effectiveness of our framework. Fig 5 shows the results of the statistical validation and examples of the rejected cases. In order to evaluate the generalization capability, we apply the same validation process to different lung HRCT data of 3 patients captured in different settings. This preliminary study resulted in $96 \%$ correct validation rate (4 false acceptances among 100 trials), similar to the results shown in Fig 5 


\section{Conclusions}

This paper proposed a robust estimation and validation framework for characterizing the location and anisotropic spread of local data structure that is approximated by a Gaussian-based model. The new framework unifies the mean shift-based robust statistical estimation and the linear scale-space-based multiscale analysis. The unification is realized by formally extending the mean shiftbased analysis towards the evaluation of continuous positive function whose local structure is characterized by an anisotropic fully-parameterized covariance matrix. The proposed statistical validation method also complements this estimation framework, providing an effective goodness-of-fit measure for rejecting accidental ill-estimates. The strength of our solution is its robustness against the margin-truncation and the non-Gaussianity effects. This advantage was demonstrated by the experimental results with the $1 \mathrm{D}$ and $2 \mathrm{D}$ synthetic data and by the $3 \mathrm{D}$ tumor analysis application.

Our proposed method can be interpreted as a multi-scale joint Gaussian fitting and segmentation. The estimation scheme achieves fitting by using only samples within the basin of attraction for characterizing the underlying structure. The importance of considering the anisotropic covariance was also suggested by Lillholm et al. [11] in their image reconstruction analyses with various local features defined as combinations of the first and second order derivatives of the scale-space representations. Their results have direct implications to our problem since the second order derivatives (or Hessian matrix) are explicitly related to the covariance matrix [13 p.178] 8 ].

The results with the real lung HRCT 3D data demonstrated a successful application of our method to the volumetric tumor analysis, providing accurate estimation of 3D location and anisotropic spread of the non-spherical pulmonary tumors. The robustness and flexibility facilitates not only the medical applications sought in this paper but also various other applications involving with the analysis of blob-like data structures. A natural continuation of this study is the extension of our framework for the automatic tumor detection problem. This remains as our future work.

Acknowledgments. The authors wish to thank Visvanathan Ramesh from Siemens Corporate Research for stimulating discussions, Alok Gupta from CAD group, Siemens Medical Solutions, for his support and encouragement, and Jonathan Stoeckel from CAD group, Siemens Medical Solutions, for his valuable technical supports. 


\section{References}

1. Y. Chen and J. McInroy. Estimating symmetric, positive definite matrices in robotic control. In IEEE Int. Conf. Robotics and Automation, pages 4269-4274, Washington D.C., 2002.

2. D. Comaniciu. An algorithm for data-driven bandwidth selection. IEEE Trans. Pattern Anal. Machine Intell., 25(2):281-288, 2003.

3. D. Comaniciu and P. Meer. Mean shift: A robust approach toward feature space analysis. IEEE Trans. Pattern Anal. Machine Intell., 24(5):603-619, 2002.

4. K. Fukunaga. Statistical Pattern Recognition. Academic Press, San Diego, 1990.

5. G. Golub and C. van Loan. Matrix Computations. Johns Hopkins University Press, Baltimore, 1996.

6. C. Henschke, D. Yankelevitz, R. Mirtcheva, G. McGuinness, and O. McCauley, D. Miettinen. CT screening for lung cancer: frequency and significance of partsolid and nonsolid nodules. AJR Am. J. Roentgenol., 178(5):1053-1057, 2002.

7. H. Hu. Positive definite constrained least-squares estimation of matrices. Linear Algebra and Its Applications, 229:167-174, 1995.

8. Y. Kanazawa and K. Kanatani. Do we really have to consider covariance matrices for image features? In Int. Conf. Computer Vision, pages 586-591, Vancouver, 2001.

9. J. Koenderink. The structure of images. Biol. Cybern., 50:363-370, 1984.

10. Y. Lee, T. Hara, H. Fujita, S. Itoh, and T. Ishigaki. Automated detection of pulmonary nodules in helical CT images based on an improved template-matching technique. IEEE Trans. Medical Imaging, 20(7):595-604, 2001.

11. M. Lillholm, M. Nielsen, and L. Griffin. Feature-based image analysis. Int. J. Comput. Vision, 52(2/3):73-95, 2003.

12. T. Lindeberg. Feature detection with automatic scale selection. Int. J. Comput. Vision, 30(2):79-116, 1998.

13. B. Matei. Heteroscedastic Errors-In-Variables Models in Computer Vision. PhD thesis, Rutgers University, 2001.

14. M. Nielsen, L. Florack, and R. Deriche. Regularization, scale space, and edge detection filters. J. Mathematical Imaging and Vision, 7(4):291-307, 1997.

15. P. Perona and J. Malik. Scale-space and edge detection using anisotropic diffusion. IEEE Trans. Pattern Anal. Machine Intell., 12(7):629-639, 1990.

16. W. Press, S. Teukolsky, W. Vetterling, and B. Flannery. Numerical Recipes in C. Cambridge University Press, Cambridge, 1992.

17. P. Rousseeuw and A. Leroy. Robust Regression and Outlier Detection. John Wiley, New York, 1987.

18. H. Takizawa, S. Yamamoto, T. Matsumoto, Y. Tateno, T. Iinuma, and M. Matsumoto. Recognition of lung nodules from X-ray CT images using 3D markov random field models. In Int. Conf. Pattern Recog., Quebec City, 2002.

19. B. van Ginneken, B. ter Harr Romeny, and M. Viergever. Computer-aided diagnosis in chest radiography: A survey. IEEE Trans. Medical Imaging, 20(12):1228-1241, 2001.

20. S. van Huffel and J. Vandewalle. The Total Least Squares Problem Computational Aspects and Analysis. SIAM, Philadelphia, 1991.

21. A. Witkin. Scale-space filtering. In Int. Joint. Conf. Artificial Intell., pages 10191021, Karlsruhe, 1983. 